\title{
Adrenal Gland and Gastric Malignant Melanoma without Evidence of Skin Lesion Treated with the Oncolytic Virus Rigvir
}

\author{
Guna Proboka a, ${ }^{a}$ Andra Tilgase ${ }^{b}$ Sergejs Isajevs ${ }^{c, d}$ Tatjana Zablockac, d \\ Evija Olmane $^{\mathrm{e}}$ Agnija Rasa $^{\mathrm{b}}$ Pēteris Alberts ${ }^{\mathrm{b}}$ \\ aLatvian Oncology Centre, Riga Eastern Clinical University Hospital, Riga, Latvia; ${ }^{6}$ Rigvir, \\ Riga, Latvia; 'Department of Pathology, Riga Eastern Clinical University Hospital, Riga, \\ Latvia; dDepartment of Pathology, Faculty of Medicine, University of Latvia, Riga, Latvia; \\ eDepartment of Radiology, Pauls Stradiňš Clinical University Hospital, Riga, Latvia
}

\section{Keywords}

Adrenal gland melanoma - Oncolytic virotherapy · Metastatic melanoma

\begin{abstract}
Adrenal gland melanoma is an extremely rare diagnosis with less than 20 cases reported. The criteria for diagnosing adrenal gland melanoma include involvement of only one adrenal gland, presence of melanin pigment in the histological examination of the tumor tissue, no primary melanoma tumor in any other organ, and no history of resection of pigmented lesions. However, it is complicated to rule out melanoma of unknown primary origin. Here we report a female patient who at the age of 75 years was admitted to hospital due to suspicion of adrenal and gastric tumor. The largest tumor was found in the adrenal gland, thus leading to the diagnosis of primary adrenal gland melanoma presenting metastases in the stomach. The melanoma was BRAF wild type. Due to the rarity of this disease, there is no standard treatment. After two subsequent surgeries, treatment with the ECHO-7 oncolytic virus Rigvir was started. The patient has received oncolytic virotherapy for 5 years and 1 month and has been stable since then with good tolerability. The therapy is still ongoing. Adrenal gland melanoma is an extremely rare diagnosis and therefore it is important to discuss the diagnostic criteria and possible treatments.


Proboka et al.: Adrenal and Gastric Melanoma Treatment with the Oncolytic Virus Rigvir

\section{Introduction}

Primary melanoma of the adrenal gland is a clinically extremely rare malignancy and only a small number of cases has been reported $[1,2]$.

Symptoms of adrenal gland melanoma are not specific; most commonly they include pain, asthenia, anorexia and weight loss, which are characteristic to any tumor process [2]. The diagnosis of primary adrenal gland melanoma is based on immunohistochemical and histopathological examination. It is not possible to observe typical tumor features from computed tomography or magnetic resonance imaging [1].

Regarding the pathogenesis of adrenal gland melanoma, its origin can be related to the neural crest. Chromaffin cells in the adrenal medulla gland originate from neural crest cells as well as melanocytes [3].

The criteria for adrenal gland melanoma diagnosis are as follows: if only one adrenal gland is involved, then the presence of melanin would be observed in the histological examination in one gland, not the other; there should be no primary melanoma tumor in any other organ, and no previously resected pigmented mucous, cutaneous or ocular lesions; the absence of hidden lesions preferably should be ruled out by autopsy results [4].

Furthermore, primary adrenal melanoma is usually a larger tumor with the largest diameter from 8 to $17 \mathrm{~cm}$, mostly observed in middle-aged and elderly people [5].

Primary malignant melanoma can arise from mucosa of the gastrointestinal tract, particularly from the esophagus, anorectum, and small bowel [6]. Less than 20 cases of primary gastric melanoma have been documented in the literature [7].

Criteria for the diagnosis of primary gastric melanoma include the absence of concurrent lesions and the lack of a history of melanoma or atypical melanocytic lesion removal from the skin or other organs $[8,9]$.

\section{Case Report}

A female patient was diagnosed at 69 years of age with adrenal gland adenoma. The patient had no complaints about her health and therefore she was only observed.

At the age of 75 years, the patient was admitted to the hospital due to suspicion of malignant adrenal and gastric tumor observed in a CT scan (Fig. 1A-E). A pathological malignant lesion in the left adrenal gland was observed. Contrast agents are not stored homogenously, suggesting the presence of necrotic zones. An enlarged lymph node in the mesentery and small lesions in the peritoneum were observed (Fig. 1D). Two months later, the malignant lesion in the left adrenal gland has increased in size with larger necrotic zones and new subcutaneous pathological lesions (Fig. 1F-J).

The extirpation of the adrenal tumor and gastrectomy were performed on September 11, 2014.

Gross examination of the adrenal specimen showed a $12.4 \times 9.7 \times 7.5 \mathrm{~cm}$ large tumor mass extending into the periadrenal tissue and metastatic to regional lymph nodes.

The histopathological examination of adrenal tumor tissue demonstrated that the tumor was composed of epitheliod and nevoid cells with large round and elongated nuclei with prominent vesicular nuclei and large nucleoli and abundant eosinophilic cytoplasm, surrounded by mature collagen bundles. The tumor cells demonstrated marked nuclear pleomorphism with variation in cell size, shape and staining, invading both through the adrenal gland, with focal hemorrhage, necrosis and lymphovascular invasion. Eight metastatic lymph nodes were revealed (Fig. 2A-C). 
Proboka et al.: Adrenal and Gastric Melanoma Treatment with the Oncolytic Virus Rigvir
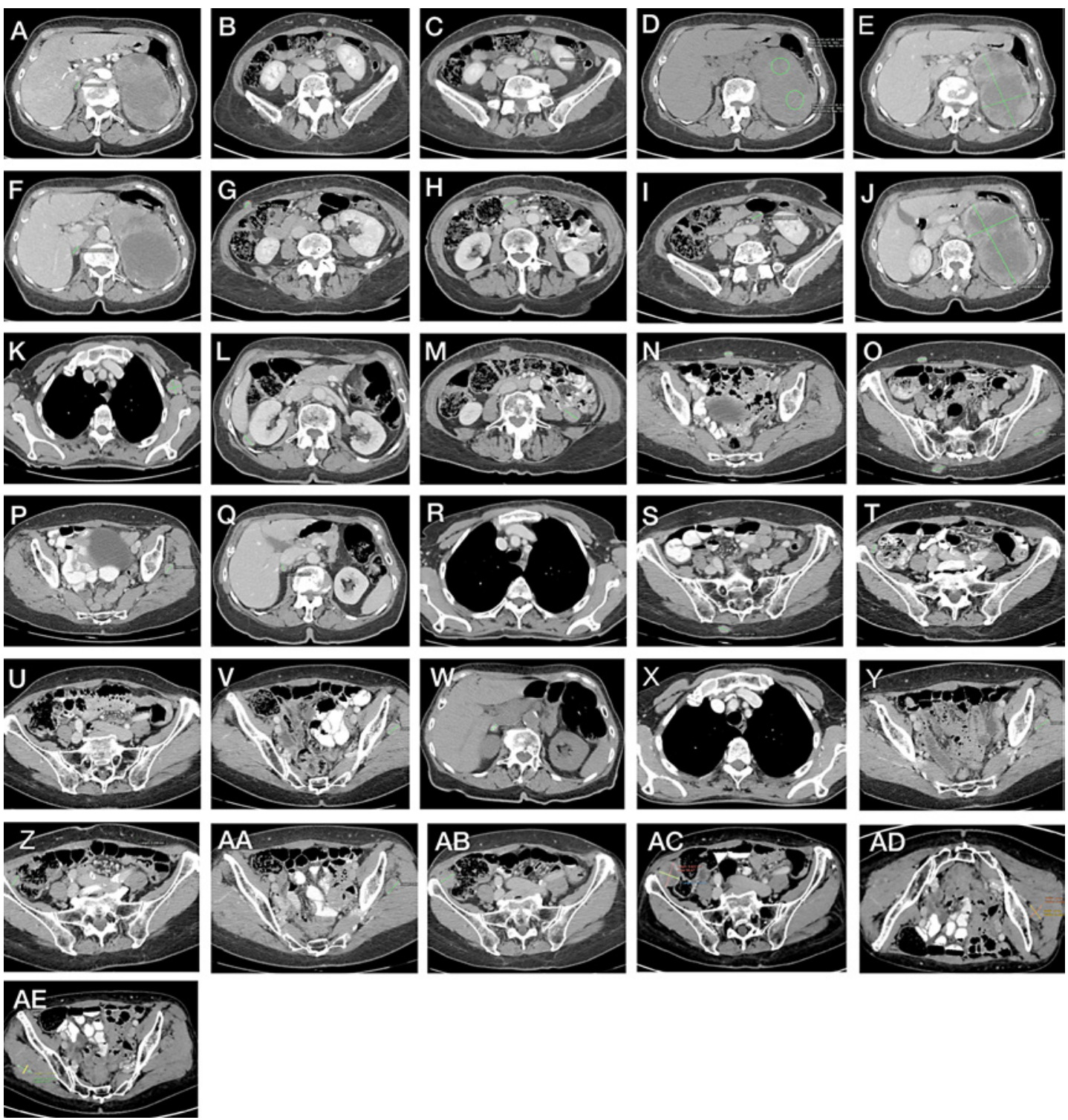

Fig. 1. Contrast-enhanced computed tomography. A-E July 2014. Pathological malignant lesion in the left adrenal gland. Contrast agents are not stored homogenously, suggesting the presence of necrotic zones. Enlarged lymph node in the mesentery and small lesions in the peritoneum were observed. F-J September 2014. The malignant lesion in the left adrenal gland has increased in size with larger necrotic zones and new subcutaneous pathological lesions. K-O February 2015. Enlarged lymph node in the axillary region, pathological lesion in the right adrenal gland, lesions in the mesenteric adipose tissue and subcutaneously as well as lesion in the left gluteal muscle were observed. P-T December 2015. Positive changes were observed; lymph node in the left axillary region has decreased in size, but lesions in the mesenteric adipose tissue and subcutaneously as well as lesion in the left gluteal muscle were still observed. U-X September 2016. Subcutaneous lesion was not observed, however, lesions in the left gluteal muscle and lesion with calcified inclusions in the right adrenal gland was observed. A new lesion laterally from the ascending colon was observed. Y, Z August 2017. No change in the radiological findings, except the lesion laterally from the ascending colon that has increased in size. AA, AB June 2018. Lesion in the left gluteal muscle and lesion laterally from the ascending colon has increased in size. AC-AE June 2019. Lesions in both gluteal regions and the right ileocecal region were observed. Lesion in the left gluteal region has increased in size by $0.3 \mathrm{~cm}$. 
Proboka et al.: Adrenal and Gastric Melanoma Treatment with the Oncolytic Virus Rigvir
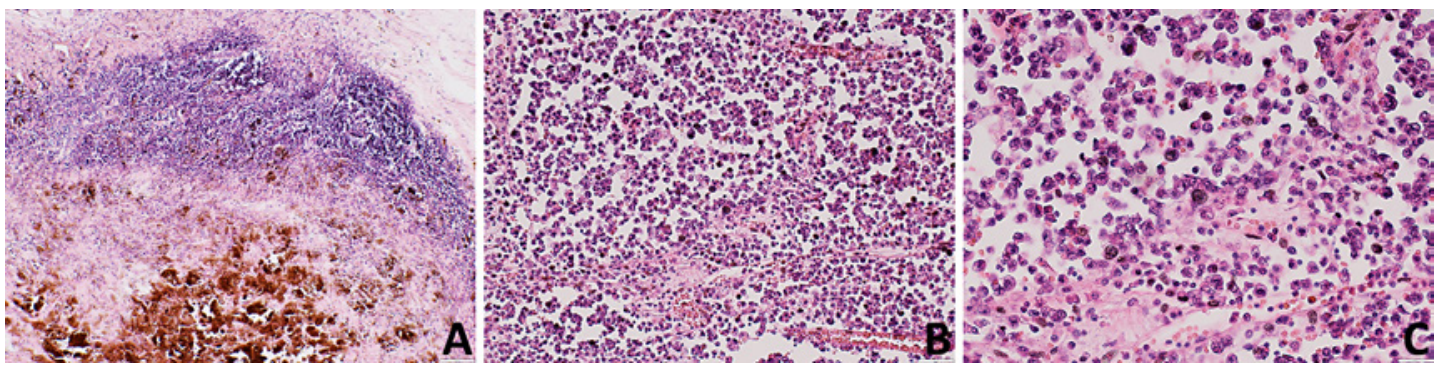

Fig. 2. Representative photomicrograph demonstrated epitheliod and nevoid pigmented melanoma metastatic to the periadrenal tissue lymph node. Magnification $\times 40(\mathbf{A})$. Nevoid and epitheliod melanoma in the adrenal gland. Magnification $\times 40(\mathbf{B})$; magnification $\times 100(\mathbf{C})$.

The adrenal tumor was Melan A-, HMB45-, S-100- and SOX-10-positive, and CKAE1/ AE3-, chromogranin- and CD56-negative.

The gross xamination demonstrated a polypoid dark grayish lesion of $7.0 \times 5.0 \mathrm{~cm}$ in the stomach. The histopathological examination of the gastric tissue showed that the tumor was composed of epitheliod and nevoid cells with large round and elongated nuclei with prominent vesicular nuclei and large nucleoli and abundant eosinophilic cytoplasm, surrounded by mature collagen bundles. The tumor cells demonstrated marked nuclear pleomorphism with variation in cell size, shape and staining. Melanin pigment was not observed by HE staining. The mitotic activity was up to 5 mitotic figures $/ \mathrm{mm}^{2}$. The tumor was located in the submucosa and muscle layers with lymphovascular invasion (Fig. 3A-D).

The gastric tumor was Melan A-, HMB45-, S-100- and SOX-10-positive, and CKAE1/AE3-, chromogranin- and CD56-negative.

A total of 14 lymph nodes were examined from the lesser omentum, two of them exhibited tumor metastasis with focal capsular invasion.

DNA from formalin-fixed paraffin-embedded gastric and adrenal melanoma tissues was isolated using GeneRead ${ }^{T M}$ DNA FFPE kit (Qiagen). The melanoma BRAF mutation status was assessed by next-generation sequencing (Qiagen).

The results show that both the gastric and adrenal gland melanoma were BRAF wild type. The patient has not had any skin or ocular lesions. The histopathological diagnosis was primary adrenal melanoma with metastases to the stomach and regional lymph nodes. Due to the extent of the disease, only symptomatic therapy was indicated.

The patient received interferon alfa-2a therapy 9 million IU, 3 times a week for a month (October 8, 2014 to November 3, 2014). However, after the interferon alfa-2a therapy, a large lymph node was detected in the armpit and the therapy was discontinued.

The patient also complained about abdominal pain and nausea. CT of the abdominal cavity was performed. Metastases in the intra-abdominal, retroperitoneal axillary region was suspected. Another lymphadenectomy was performed, and three additional lymph nodes were excised on November 10, 2014.

After 5 months, an enlarged lymph node in the axillary region, a pathological lesion in the right adrenal gland, lesions in mesenteric adipose tissue and subcutaneously as well as a lesion in the left gluteal muscle were observed (Fig. 1K-0).

On January 6, 2015, the patient started Rigvir therapy. Three injections were administered on 3 consecutive days and then 1 injection in 2 weeks was administered. The therapy is still ongoing.

After 11 months, positive changes were observed; the lymph node in the left axillary region has decreased in size, while lesions in the mesenteric adipose tissue and subcutane- 
Proboka et al.: Adrenal and Gastric Melanoma Treatment with the Oncolytic Virus Rigvir
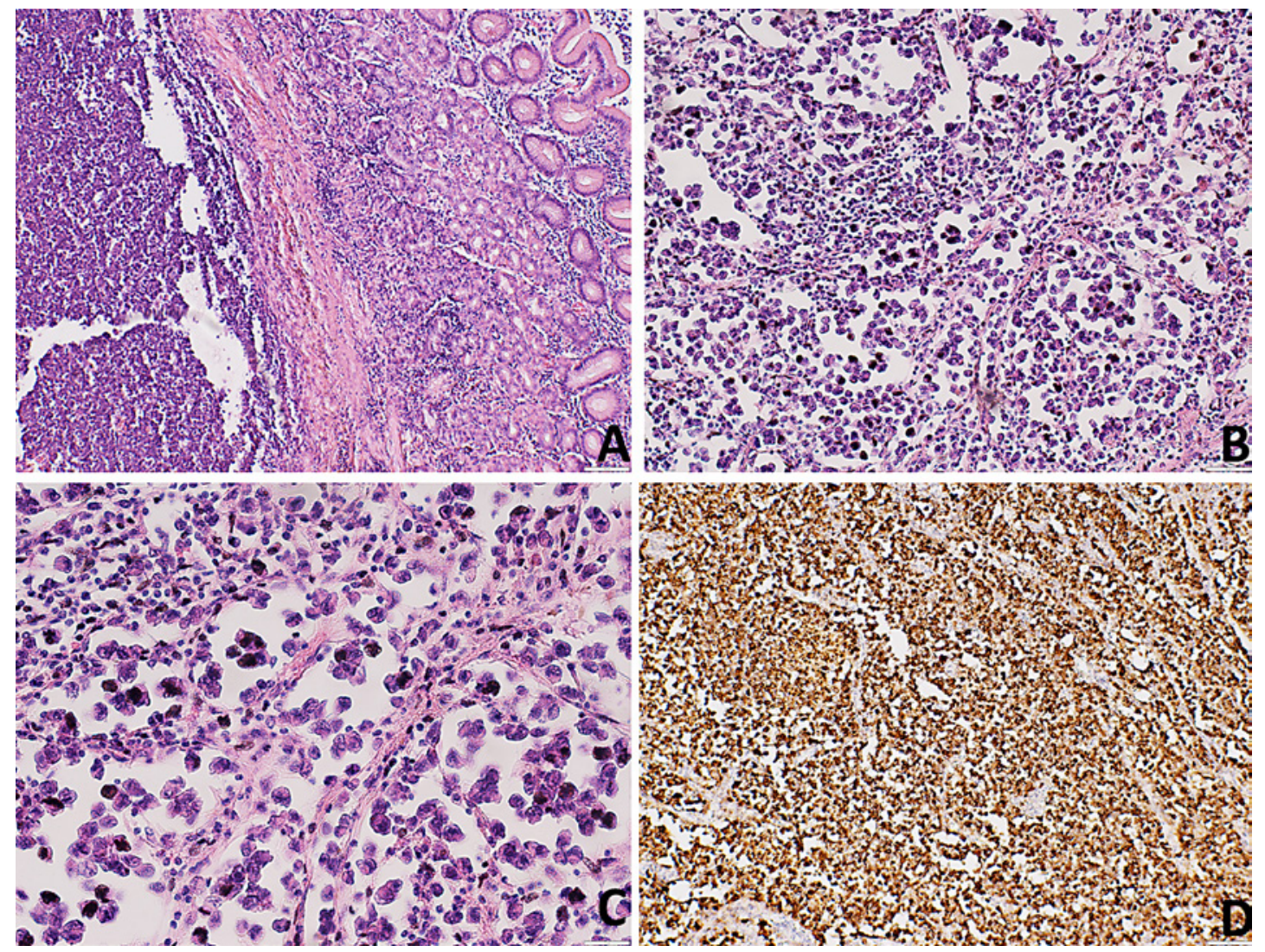

Fig. 3. Representative photomicrographs demonstrated epitheliod and nevoid weakly pigmented melanoma in the gastric submucosa and muscle layer tissue. Magnification $\times 40($ A); magnification $\times 100$ (B); magnification $\times 200$ (C). Immunohistochemical staining demonstrated Melan A expression in the tumor, magnification $\times 100$ (D), scale bar $=100 \mu \mathrm{m}$.

ously and the lesion in the left gluteal muscle were still observed (Fig. 1P-T). In September 2016, no subcutaneous lesion was observed, however, lesions in the left gluteal muscle and a lesion with calcified inclusions in the right adrenal gland were observed. A new lesion laterally from the ascending colon was observed (Fig. 1U-X). In August 2017, no change in the radiological findings was observed, except the lesion laterally from the ascending colon that had increased in size (Fig. 1Y-Z). In June 2018, the lesion in the left gluteal muscle and the lesion laterally from the ascending colon had increased in size (Fig. 1AA-AB). In June 2019 , the lesion in the left gluteal muscle and the lesion laterally from the ascending colon had increased in size by $0.3 \mathrm{~cm}$. There was no evidence of a malignant process in the thoracic area.

\section{Discussion}

Adrenal gland melanoma is a rare disease with few cases described. In a recent case report and literature review, it has been stated that only 14 cases of adrenal melanoma have been reported corresponding to Carsten's criteria to this day [1].

It is well known that melanoma is an aggressive malignancy with a tendency to metastasize fast to other parts of the body, including the adrenal glands. 
Proboka et al.: Adrenal and Gastric Melanoma Treatment with the Oncolytic Virus Rigvir

The diagnostic criteria of primary adrenal melanoma are that only one adrenal gland is involved, that skin or visceral melanoma have been excluded, with no previous surgical history of pigmented mucous, cutaneous, or ocular lesions $[4,10]$. The criteria for primary gastric melanoma are similar, for example, absence of concurrent lesions and the lack of a history of melanoma or atypical melanocytic lesion removal from the skin or other organs $[8,9]$.

Concerning the origin, melanoma is considered to be a heterogeneous entity, comprising of cases with fully regressed primary lesions, cases of internal or nodal primary melanomas, and cases where the primary tumor had been treated with nonsurgical procedures or excised without proper histological confirmation [11].

Our study demonstrates a challenging case of melanoma in the adrenal gland and stomach without a clinical history of any skin and ocular lesions. Both tumors were BRAF wild type. At present, there are not enough criteria available to distinguish between these two types of cancers, especially considering their rarity. Melanoma of unknown primary with metastasis in the adrenal gland, stomach and lymph nodes could also not be excluded.

Melanoma of unknown primary or occult primary melanoma represents only a minority of melanoma cases, occurring in almost 3\% of patients [12]. The diagnosis of melanoma of unknown primary is commonly based on the lack of proof of a primary cutaneous, ocular or mucosal lesion.

Currently, only Carsten's criteria are used to diagnose adrenal gland melanoma. With the given criteria, it is complicated to distinguish the diagnosis since no specific marker for adrenal melanoma is used in the current diagnostic process.

Considering the rarity of adrenal gland melanoma, there is no standard treatment. The present patient experienced a disease progression after 2 months of interferon alfa-2a therapy and that treatment was discontinued. Subsequently, the patient started oncolytic virotherapy with Rigvir. Since then, disease progression has slowed down and the patient has been stable for 5 years. No adverse events have been reported by the patient.

In conclusion, considering the lack of standard treatment for adrenal gland melanoma and the good tolerability of oncolytic virus Rigvir treatment, the use of Rigvir deserves to be investigated in more detail for the treatment for adrenal gland melanoma.

\section{Statement of Ethics}

The patient has given her written informed consent to publish her case (including the publication of images).

Off-label has been defined as "all uses of a marketed drug not detailed in the summary of product characteristics including therapeutic indication, use in age-subsets, appropriate strength (dosage), pharmaceutical form and route of administration" [13], and a "different indication in term of medical condition than the one described in the authorised product information; a different group of patients than the one described in the authorised product information; a different route or method of administration than the one described in the authorised product information; a different posology than the one described in the authorised product information" [14]. The off-label use in oncology for adults has been reported to be in the range of 10-76\%, depending on the cancer [15]. Consequently, in the case reported here, the off-label use is not part of a prospective study but part of daily practice and as such does not require the approval of an ethics committee. 
Proboka et al.: Adrenal and Gastric Melanoma Treatment with the Oncolytic Virus Rigvir

\section{Disclosure Statement}

G.P., A.T., A.R and P.A. are employees of Rigvir. E.O., S.I. and T.Z. have no conflicts of interest to declare.

\section{Funding Sources}

Not applicable.

\section{Author Contributions}

G.P. is the attending oncologist and gathered the data, A.T. analyzed data and drafted the manuscript, S.I. and T.Z. performed histological analyses, E.O. analyzed radiological data, A.R. analyzed the data and reviewed the manuscript, P.A. substantially contributed to the conception of the work critically and revised the manuscript.

\section{References}

1 Xu B, Hong Y, Jin M, Li M, Wang C, Wang X. Primary adrenal malignant melanoma: A case report and review of literature. Medicine (Baltimore). 2017;96(51):e8956.

2 González-Sáez L, Pita-Fernández S, Lorenzo-Patiño MJ, Arnal-Monreal F, Machuca-Santacruz J, RomeroGonzález J. Primary melanoma of the adrenal gland: a case report and review of the literature. J Med Case Rep. 2011;5:273.

3 Tanas MR, Rubin BP. Malignant neuroectodermal tumor with melanocytic and rhabdomyoblastic differentiation. Rare Tumors. 2009;1(2):e26.

4 Carstens PH, Kuhns JG, Ghazi C. Primary malignant melanomas of the lung and adrenal. Hum Pathol. 1984; 15(10):910-4.

5 Granero LE, Al-Lawati T, Bobin JY. Primary Melanoma of the Adrenal Gland, a Continuous Dilemma: Report of a Case. Surg Today. 2004;34(6):554-6.

6 Aagaard MT, Kristensen IB, Lund O, Hasenkam JM, Kimose HH. Primary Malignant Non-Epithelial Tumours of the Thoracic Oesophagus and Cardia in a 25-Year Surgical Material. Scand J Gastroenterol. 1990;25(9):87682.

7 Chandler AB, Jones GF. Malignant melanoma of the gastrointestinal tract; a case report. Am Surg. 1951;17(8): 719-21.

8 Elsayed AM, Albahra M, Nzeako UC, Sobin LH. Malignant melanomas in the small intestine: a study of 103 patients. Am J Gastroenterol. 1996;91(5):1001-6.

9 Simons M, Ferreira J, Meunier R, Moss S. Primary versus Metastatic Gastrointestinal Melanoma: A Rare Case and Review of Current Literature. Case Rep Gastrointest Med. 2016;2016:2306180.

10 Ainsworth AM, Clark WH Jr, Mastrangelo M, Conger KB. Primary malignant melanoma of the urinary bladder. Cancer. 1976;37(4):1928-36.

11 Savoia P, Fava P, Osella-Abate S, Nardò T, Comessatti A, Quaglino P, et al. Melanoma of unknown primary site: a 33-year experience at the Turin Melanoma Centre. Melanoma Res. 2010;20(3):227-32.

12 Kamposioras K, Pentheroudakis G, Pectasides D, Pavlidis N. Malignant melanoma of unknown primary site. To make the long story short. A systematic review of the literature. Crit Rev Oncol Hematol. 2011;78(2):112-26.

13 Neubert A, Wong IC, Bonifazi A, Catapano M, Felisi M, Baiardi P, et al. Defining off-label and unlicensed use of medicines for children: Results of a Delphi survey. Pharmacol Res. 2008;58(5-6):316-22.

14 EMA. Reflection paper on collecting and reporting information on off-label use in pharmacovigilance. 2016. Available from: https://www.ema.europa.eu/en/documents/regulatory-procedural-guideline/reflectionpaper-collecting-reporting-information-label-use-pharmacovigilance_en.pdf.

15 Weda M HJ, Vervolet M, et al. Study on off-label use of medicinal products in the European Union. 2017. Available from: https://ec.europa.eu/health/sites/health/files/files/documents/2017_02_28_final_study_ report_on_off-label_use_pdf. 